\title{
EFFECTS OF COLLISIONS WITH ROCKY PLANETS ON THE PROPERTIES OF HOT JUPITERS
}

\author{
Kassandra R. Anderson ${ }^{1}$ and Fred C. Adams ${ }^{1,2}$ \\ ${ }^{1}$ Physics Department, University of Michigan, Ann Arbor, MI 48109 \\ ${ }^{2}$ Astronomy Department, University of Michigan, Ann Arbor, MI 48109
}

\begin{abstract}
Observed Hot Jupiters exhibit a wide range of physical properties. For a given mass, many planets have inflated radii, while others are surprisingly compact and may harbor large central cores. Motivated by the observational sample, this paper considers possible effects from collisions of smaller rocky planets with gas giant planets. In this scenario, the Jovian planets migrate first and enter into $\sim 4$ day orbits, whereas rocky planets (mass $m_{\mathrm{p}}=0.1-20 M_{\oplus}$ ) migrate later and then encounter the gaseous giants. Previous work indicates that the collision rates are high for such systems. This paper calculates the trajectories of incoming rocky planets as they orbit within the gaseous planets and are subjected to gravitational, frictional, and tidal forces. These collisions always increase the metallicity of the Jovian planets. If the incoming rocky bodies survive tidal destruction and reach the central regions, they provide a means of producing large planetary cores. Both the added metallicity and larger cores act to decrease the radii of the gas giants at fixed mass. The energy released during these collisions provides the Jovian planet with an additional heat source; here we determine the radial layers where kinetic energy of the colliding body is dissipated, including the energy remaining upon impact with the existing core. This process could have long-term effects if the colliding body deposits significant energy deep in the interior, in regions of high opacity. Both Hot Jupiters and newly formed gas giants have inflated radii, large enough to allow incoming rocky planets to survive tidal disruption, enhance the central core mass, and deposit significant energy (in contrast, denser giant planets with the mass and radius of Jupiter are expected to tidally destroy incoming rocky bodies).
\end{abstract}

Subject headings: planets and satellites: composition — planets and satellites: dynamical evolution and stability — planets and satellites: formation 


\section{Introduction}

Since the first discovery of an exoplanet orbiting a main-sequence star (Mavor \& Queloz 1995), the field of extrasolar planets has undergone rapid progress. More than 750 planets have been discovered beyond our Solar System, with over 1000 more possible objects awaiting further measurements for confirmation (Borucki et al. 2011). Although the observational sample is far from complete, it is clear that the Galaxy hosts a diverse population of planets, with a range of masses spanning over three orders of magnitude. More than 150 of the $\sim 750$ confirmed planets are gas giants with semi-major axes less than $0.1 \mathrm{AU}$. These planets are thought to reach such extreme environments after forming further out in their circumstellar disks and migrating inward (Papaloizou \& Terquem 2006). Up to $\sim 5 \%$ of all solar systems are believed to harbor "Hot Jupiters" (Bramich \& Horne 2006; van Saders \& Gaudi 2011), so that a billion such planets could reside in the Galaxy.

The observed Hot Jupiters have a wide range of radii for a given mass (Baraffe et al. 2010; Burrows \& Orton 2009). Many planets are larger than predicted, but some are surprisingly small. The reasons for these anomalous radii remain uncertain, and the construction of a definitive mass-radius theory for Hot Jupiters has become a central problem in exoplanet research (e.g., Bodenheimer et al. 2003; Laughlin et al. 2011). In general terms, the inflated planets can be understood if they contain additional energy sources (Bodenheimer et al. 2003; Gu et al. 2004), e.g., heat generated by Ohmic dissipation (Batygin \& Stevenson 2010; Perna et al. 2010; Batvgin et al. 2011), conversion of wind kinetic energy into heat (Rauscher \& Menou 2010; Guillot \& Showman 2002), and/or tidal dissipation (Arras \& Socrates 2010). In contrast, giant planets with deflated radii must have high metallicities (Miller \& Fortney 2011) and possibly large cores (Burrows et al. 2007). The problem of these anomalous radii thus splits into two sub-problems: [a] the source(s) of additional heating, and [b] the ways in which planets can accumulate metallicity and large cores. Identifying possible long-term heat sources and examining the ways in which planets can increase their core masses will help explain the observed mass-radius relation and increase our understanding of giant planet formation, structure, and evolution.

The goal of this paper is relatively modest: We consider the effects of collisions of smaller rocky bodies with gaseous giant planets, and explore how the collisions affect metallicity, core size, and perhaps the energy budget. During the early epochs of solar system formation, a large number of small terrestrial bodies are present; previous work has shown that they can migrate inward and become captured by a Hot Jupiter with high probability (Ketchum et al. 2011b). After capture, a rocky planet orbiting within the interior of a Hot Jupiter loses energy due to dissipative forces and eventually either collides with the core or becomes tidally disrupted. These impacts provide a natural mechanism for increasing the 
planetary metallicity, and could help explain some of the inferred heavy cores and metal-rich compositions of the Hot Jupiters. In addition, a substantial amount of energy is released during these collisions. This energy could inflate a giant planet over a longer period of time, provided that the energy is deposited deep within the planet interior where the opacity is high. On the other hand, if the kinetic energy from the collision is dissipated in the outer planetary layers, it would provide only a short-term source of energy. These impacts thus have the potential to help explain, at least in part, the observed radius anomalies.

Given the strong observational evidence for planets with large cores, the process of core enhancement is of particular interest. A specific observational example is provided by the planet HD $149026 \mathrm{~b}$, with a total mass $M_{P}=0.36 \pm 0.03 M_{J}$ and a radius $R_{P}=$ $0.73 \pm 0.03 R_{J}($ Sato et al. 2005). These observational constraints, along with theoretical calculations (Fortney et al. 2006; Ikoma et al. 2006), indicate that this planet contains $\sim 80$ $M_{\oplus}$ of heavy elements. A significant fraction of these heavy elements could reside in the core. Although current observations do not fully constrain the core mass, it is expected that HD $149026 \mathrm{~b}$ and similar planets have larger cores than predicted by theories of planet formation. According to the core accretion model (Pollack et al. 1996), giant planet cores are formed from rocky planetesimals in the circumstellar disk, and begin to accrete gas after reaching a critical mass around $10 M_{\oplus}$. Sufficiently large planets experience runaway gas accretion and clear gaps in their disks, so that the accretion of additional rocky material is suppressed, making it difficult to build up core masses as large as $80 M_{\oplus}$. The planet HD 149026b, and others, motivates the possible role of collisions in shaping giant planet cores. A gaseous planet with an initially small core mass could accrete one or more rocky planets early in its lifetime and thereby accumulate a heavier core. Collisions with superearths $\left(m_{\mathrm{p}} \approx 10 M_{\oplus}\right)$ are probably the best way to explain the most massive cores, since the accumulation of a large core from low-mass planets would require a high encounter rate. In addition, small planets are unlikely to survive tidal forces over the entire trajectory and reach the core. However, the accretion of smaller planets could affect the structure of the giant planets by increasing the metallicity of the envelope.

In this paper we model the collisional dynamics between Jovian planets and smaller rocky planets. This problem has been explored previously in the context of Jupiter and Saturn (Anic et al. 2007; Li et al. 2010) using smooth particle hydrodynamical (SPH) simulations. These authors have investigated both the effects on the overall structure of the giant planet and survival of the colliding bodies. The conditions required for rocky planets to survive are particularly important. The previous results indicate that low-mass planets $\left(m_{\mathrm{p}} \approx 1 M_{\oplus}\right)$ are often destroyed prior to reaching the core, whereas larger planets $\left(m_{\mathrm{p}} \approx 10 M_{\oplus}\right)$ usually survive the entire trajectory. This paper extends this previous work, but adopts a complementary approach. SPH (or equivalent) methods provide a detailed de- 
scription of the collisions, but are computationally expensive and allow relatively few cases to be considered. Here, instead, we treat the collision as a one-body problem, where the incoming rocky body orbits within the giant planet and is subject to gravitational, frictional, and tidal forces. This approach allows for a much wider survey of parameter space, which, in spite of the simplifying approximations, increases our physical understanding of these collisions. More specifically, this paper investigates the accretion of rocky bodies spanning two orders of magnitude in mass $\left(m_{\mathrm{p}}=0.1-20 M_{\oplus}\right)$, within gas giant planets of varying properties. Here we focus on Jovian planets with mass $M_{P}=1 M_{J}$ and radii in the range $R_{P}=1-2 R_{J}$, where the latter are appropriate for young Hot Jupiters (Burrows et al. 1997).

This paper is organized as follows: In Section 2, we construct polytropic models for gas giant planets both with and without central cores. Using this model in Section 3, we calculate the trajectories of incoming rocky bodies within the gaseous giant planets. These results determine whether the rocky body is captured or passes through the giant planet (as a function of initial speed and impact parameter). These trajectories also specify the radial locations where energy is dissipated and the kinetic energy remaining upon impact with the existing core. In Section 4, we calculate the tidal forces exerted on an incoming rocky planet by its host Jovian planet; such tidal effects are a function of radial distance and determine whether or not the rocky planet can survive. We conclude, in Section 5 , with a summary of our results and a discussion of their implications.

\section{Planetary Structure Models}

Before considering planetary collisions, we develop a structure model for the Jovian (target) planet. Here we assume that the Jovian planet consists of a rocky core of constant density surrounded by a gaseous envelope. The planet is assumed to be in hydrostatic equilibrium, and obeys the equations of hydrostatic equilibrium and mass conservation,

$$
\frac{d P}{d r}=-\frac{G M(r) \rho(r)}{r^{2}} \quad \text { and } \quad \frac{d M}{d r}=4 \pi r^{2} \rho(r) .
$$

After specifying the equation of state $P(\rho)$, these equations can be combined into a single differential equation, which can be solved for the density profile. The conditions within Jovian planets require complicated equations of state, which can vary from planet to planet. Since the goal of this paper is to develop a relatively simple model to survey the parameter space for collisions, we adopt a polytropic equation of state of the form

$$
P=K \rho^{\Gamma} \quad \text { where } \quad \Gamma=1+\frac{1}{n},
$$


and where $n$ is the polytropic index. Here we consider a range of polytropic indices $n=1-2$, but detailed models suggest Jovian planets are characterized by indices near the lower end $(n \sim 1)$ of this range (e.g., Burrows 2011). As shown below, the mean density of the planet is one of the most important physical variables in the problem; for polytropic models with fixed mass and radius, the mean density does not differ greatly from that of more detailed models. Further, the system to system variations in the structure of observed exoplanets is larger than the differences resulting from this polytropic approximation.

Following the formulation of Chandrasekhar (1939), we define dimensionless quantities $\xi$ and $f(\xi)$ through the relations

$$
\xi=\frac{r}{R} \quad \text { and } \quad \rho=\rho_{c} f^{n}(\xi), \quad \text { where } \quad R^{2}=\frac{K \Gamma}{(\Gamma-1) 4 \pi G \rho_{c}^{2-\Gamma}} .
$$

With these definitions, the resulting differential equation for $f(\xi)$ becomes the Lane-Emden equation

$$
\frac{1}{\xi^{2}} \frac{d}{d \xi}\left(\xi^{2} \frac{d f}{d \xi}\right)+f^{n}=0
$$

with the boundary conditions

$$
f\left(\xi_{c}\right)=1 \quad \text { and }\left.\quad \frac{d f}{d \xi}\right|_{\xi=\xi_{c}}=0,
$$

where $\xi_{c}$ denotes the radius of the planetary core. Planets without central cores correspond to the limit $\xi_{c} \rightarrow 0$. The first zero of the function $f(\xi)$, denoted here at $\xi_{0}$, marks the outer boundary (the surface) of the planet.

Although polytropic stellar structure models have been used extensively (Chandrasekhar 1939; Phillips 1994), this application differs from most previous cases in two respects: First, we include the possibility of a constant density core, so that the (dimensionless) core radius $\xi_{c}$ provides an additional parameter. Second, the expected values of the polytropic index are closer to $n=1$ for giant planets, instead of the more familiar value $n=3 / 2$ for degenerate stars and convective stars, or $n=3$ for massive stars dominated by radiation pressure (Phillips 1994).

In terms of the dimensionless variables, the mass conservation equation is given by

$$
\frac{d \mu}{d \xi}=\xi^{2} f^{n}
$$

which is related to the physical enclosed mass profile $M(r)$ via

$$
M(r)=4 \pi R^{3} \rho_{c} \mu(\xi)
$$


The total dimensionless mass $\mu_{0}$ of the planet is thus

$$
\mu_{0}=\int_{0}^{\xi_{0}} \xi^{2} f^{n}(\xi) d \xi
$$

and the physical mass and radius are given by

$$
M_{P}=4 \pi R^{3} \rho_{c} \mu_{0} \quad \text { and } \quad R_{P}=R \xi_{0} .
$$

Although the planetary structure is hydrostatic and does not require a time variable, orbital motion is time dependent. Here we define a dimensionless time variable

$$
\tau=\frac{t}{t_{0}} \quad \text { where } \quad t_{0}=\left(4 \pi G \rho_{c}\right)^{-1 / 2} .
$$

The time scale $t_{0}$ is the free-fall time appropriate for the density at the planet center, and provides a benchmark dynamical time scale for the orbit calculations. As an order of magnitude estimate, the central densities of Jovian planets $\rho_{c} \sim 10 \mathrm{~g} \mathrm{~cm}^{-3}$, which corresponds to a time scale $t_{0} \sim 345 \mathrm{sec} \sim 6 \mathrm{~min}$.

Numerical integration of equation (44) yields a dimensionless density profile, which can be converted to physical units by specifying the central density. The surface of the planet corresponds to the radius $\xi_{0}$ where the density and pressure vanish. Solutions for $f(\xi)$ are shown in Figure 1 for a polytropic index $n=1$ and dimensionless core radii $\xi_{c}=0-1$. Variation of the parameters $\xi_{c}$ and $n$ lead to a range of density profiles, and upon specification of the physical planet mass and radius, different central densities and pressures, core masses, and core radii. This model thus allows us to explore a variety of planet structures. For this paper, we vary the core radius over a wide range, with a focus on $\xi_{c}=0-1$, and vary the polytropic index over the range $n=1-2$. For planets with Jovian masses and radii, we obtain central densities $\rho_{c}=2-15 \mathrm{~g} \mathrm{~cm}^{-3}$, leading to central pressures of order $100 \mathrm{Mbar}$, in agreement with more complicated models (e.g., Militzer et al. 2008). For a dimensionless core radius $\xi_{c}=1$, the core mass (in physical units) thus has values $m_{c} \approx 20-30 M_{\oplus}$ (for varying $n$ ).

In general, equation (4) must be numerically integrated. For the particular case where the polytropic index $n=1$, however, the Lane-Emden equation (44) has an analytic solution of the form

$$
f(\xi)=A \frac{\cos \xi}{\xi}+B \frac{\sin \xi}{\xi} .
$$

For a coreless polytrope, the first term must vanish to avoid divergence at the origin $(A=0)$, and the solution becomes

$$
f(\xi)=\frac{\sin \xi}{\xi}
$$




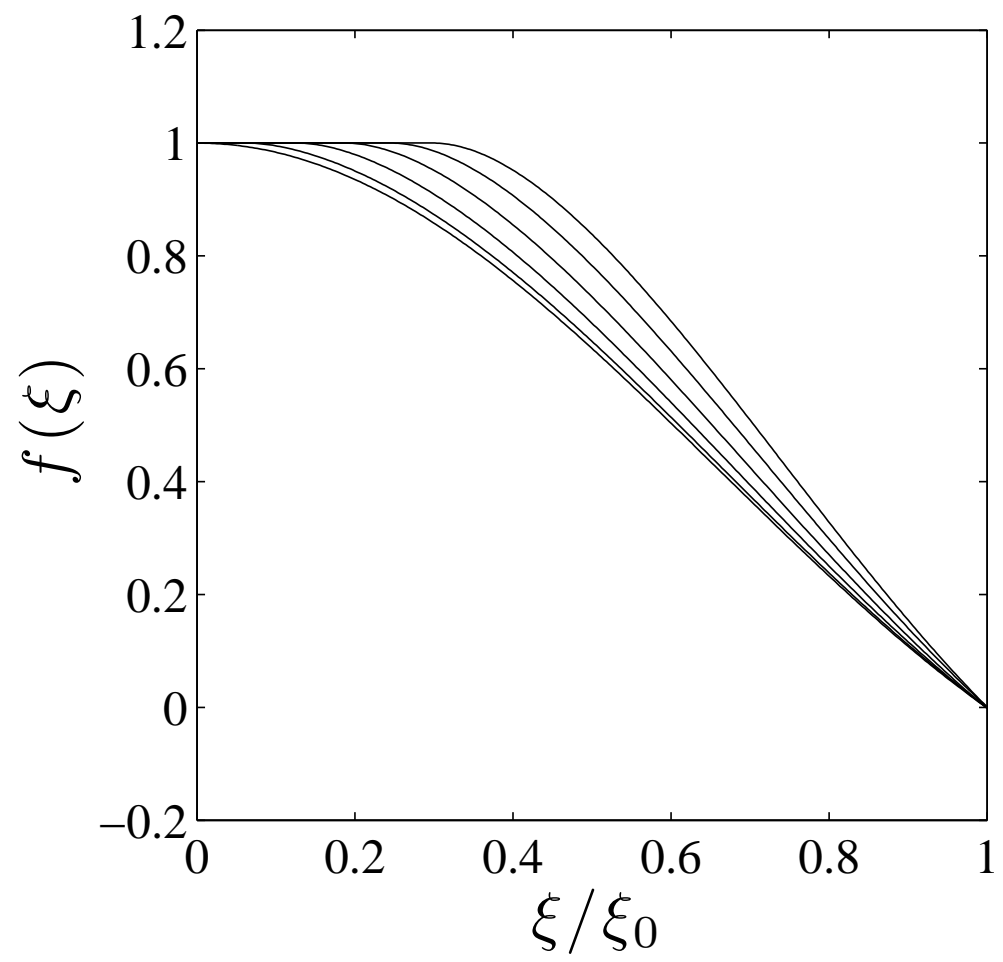

Fig. 1.- Density profiles for $n=1$ polytropic models with a collection of core masses. Each curve corresponds to a different core radius from $\xi_{0}=0$ (bottom) to $\xi_{c}=1$ (top). For the largest value shown, $\xi_{c}=1$, the core makes up $\sim 6.6 \%$ of the mass (e.g., the core would have mass $m_{c} \sim 21 M_{\oplus}$ for a giant planet mass $M_{P}=1 M_{J}$ ). 
Note that the first zero occurs at $\xi_{0}=\pi$. For finite core radii $\left(\xi_{c} \neq 0\right)$, the general solution takes the form

$$
f(\xi)=\left(\xi_{c} \cos \xi_{c}-\sin \xi_{c}\right) \frac{\cos \xi}{\xi}+\left(\xi_{c} \sin \xi_{c}+\cos \xi_{c}\right) \frac{\sin \xi}{\xi} .
$$

Note that for this general case, the first zero $\xi_{0}$ must be determined by solving a transcendental equation. However, analytic expressions for the mass profiles $\mu(\xi)$ can be found by substituting these solutions for $f(\xi)$ into equation (6). For the coreless polytrope, we find

$$
\mu(\xi)=-\xi \cos \xi+\sin \xi
$$

For the general case, $\mu(\xi)=\xi^{3} / 3$ for $\xi \leq \xi_{c}$, and

$$
\mu(\xi)=\frac{1}{3} \xi_{c}{ }^{3}+\left(\xi_{c}-\xi\right) \cos \left(\xi-\xi_{c}\right)+\left(1+\xi_{c} \xi\right) \sin \left(\xi-\xi_{c}\right)
$$

for $\xi>\xi_{c}$. Although the coreless polytrope represents a relatively good model for planets with small cores like Jupiter, it is less accurate for the exoplanets with massive cores. Nonetheless, it provides a reasonable approximation and the analytic forms for the density and mass profiles are convenient for later calculations.

For a coreless polytrope of fixed index $n$, the model provides a two parameter family of solutions, where the parameters are the central density $\rho_{c}$ and the coefficient $K$ of the equation of state (note that $K$ depends on the physics of the planetary interior and can take a range of values). For any planet with observed mass $M_{P}$ and radius $R_{P}$, we can (in principle) adjust $\rho_{c}$ and $K$ to provide the correct properties. This procedure results in a prediction for the central density $\rho_{c}$ and the central pressure $P_{c}=K \rho_{c}^{\Gamma}$. As a consistency check, we fit the model to a planet with the mass and radius of Jupiter, calculate the central pressure and density, and then compare with current estimates. For example, a coreless model with $n=1$ produces a central density $\rho_{c} \approx 4 \mathrm{~g} \mathrm{~cm}^{-3}$ and a corresponding central pressure pressure $P_{c} \approx 40$ Mbar. The actual central properties of Jupiter are somewhat uncertain because the interior cannot be observed directly and the conditions are difficult to recreate experimentally. However, the central pressure of Jupiter is thought to lie in the range $P_{c}$ $=10-100 \mathrm{Mbar}$, with estimates around $P_{c} \approx 70 \mathrm{Mbar}$ (Saumon \& Guillot 2004), in rough agreement with our predicted model values. More detailed knowledge of these conditions requires a reliable equation of state for hydrogen at high pressures, especially in the transition to metallic hydrogen. This poses significant experimental and theoretical challenges, and many uncertainties remain regarding the interiors of gas giants (Hubbard et al. 2002).

The central pressure of the planet must obey the relation

$$
P_{c}=\mathcal{F} \frac{G M_{P}^{2}}{\pi R_{P}^{4}}=K \rho_{c}^{\Gamma} .
$$


The first expression follows from the condition of hydrostatic equilibrium (and dimensional analysis) whereas the second follows from the polytropic equation of state. The dimensionless parameter $\mathcal{F}$ is expected to be of order unity and can be written in the form

$$
\mathcal{F}=\frac{\xi_{0}^{4}}{4 \mu_{0}^{2}(n+1)} .
$$

For an $n=1$ polytrope with no core, $\xi_{0}=\pi=\mu_{0}$, so that $\mathcal{F}=\pi^{2} / 8$. As the core size increases, the factor $\mathcal{F}$ decreases, so that $\mathcal{F} \approx 0.242$ for a core with $\xi_{c}=2$. For larger values of polytropic index $n$, the factor $\mathcal{F}$ is larger for planets with no core, but has nearly the same values for planets with large central cores.

\section{Orbital Trajectories}

In this section we solve the equations of motion for a rocky planet as it falls into a gaseous giant planet. In this setting, we assume that the Hot Jupiter has completed its inward migration and entered into a stable orbit close to its star. Rocky planets migrate in later and have a high probability of colliding with the Hot Jupiter, provided that their

orbital eccentricity is not overly damped (Ketchum et al. 2011b). At the start of these integrations, the rocky planet already lies well within the Hill sphere of the Jovian planet so that the gravitational field of the star can be neglected. The system thus consists of a Jovian planet with a mass $M_{P}=1 M_{J}$ and a smaller rocky planet with mass $m_{\mathrm{p}} \ll M_{P}$. To first approximation, the system can be treated as a one-body problem, with the origin of the coordinate system placed at the center of the Jovian planet. As the rocky planet orbits within the gaseous envelope of the Jovian planet, it experiences the usual gravitational force and a frictional (ram pressure) force, so that the equation of motion is given by

$$
\ddot{\mathbf{r}}=-\frac{G M(r)}{r^{2}} \hat{r}-\frac{\rho(r) v^{2} \Sigma_{\mathrm{p}}}{m_{\mathrm{p}}} \hat{v},
$$

where $\Sigma_{\mathrm{p}} \sim \pi r_{\mathrm{p}}{ }^{2}$ is the effective cross sectional area of the rocky planet (for determining ram pressure forces). Using definitions from equation (3), we obtain

$$
\frac{\ddot{\mathbf{r}}}{R}=\ddot{\vec{\xi}}=-\frac{\mu(\xi)}{\xi^{2}} \hat{r}-\alpha f^{n} v^{2} \hat{v},
$$

where $v$ is now the dimensionless speed (in units of $R / t_{0}$ ), and where time derivatives are performed with respect to the dimensionless time $\tau$ (from equation [10]). We have also defined a dimensionless friction coefficient

$$
\alpha \equiv \frac{\xi_{0}^{2}}{4 \pi \mu_{0}}\left(\frac{M_{P}}{m_{\mathrm{p}}}\right)\left(\frac{\Sigma_{\mathrm{p}}}{R_{P}{ }^{2}}\right) .
$$


Note that the coefficient $\alpha$ depends on the masses and radii of both planets, as well as the polytropic index and the core radius. Here we consider rocky planets with masses $m_{\mathrm{p}}=0.1-20 M_{\oplus}$. For a typical mean density $\langle\rho\rangle=5.5 \mathrm{~g} \mathrm{~cm}^{-3}$ for the rocky planet, and for $M_{P}=M_{J}$ and $R_{P}=R_{J}$, the corresponding values of the coefficient $\alpha$ lie in the range $\alpha \approx 0.5-5$. Note that Jovian planets with inflated radii have lower values of $\alpha$ and provide less friction.

The relevant parameter space thus consists of five variables: the impact parameter $x_{0}$ of the collision, initial velocity $v_{0}$, friction coefficient $\alpha$, the polytropic index $n$, and the dimensionless core radius $\xi_{c}$. Once these parameters are specified, the equation of motion (19) can be numerically integrated.

\subsection{Rocky Planets Orbiting within Jovian Planets}

To begin, we consider the simplest case of a coreless Jovian planet $\left(\xi_{c}=0\right)$ and adopt a polytropic index $n=1$. By fixing the polytropic index and core radius, the number of free parameters is reduced to three. The starting conditions are defined as follows: The calculations are started with the incoming rocky planets located at the outer edge of the Jovian planet, traveling in the $-\hat{y}$ direction. In dimensionless coordinates, the rocky planet has initial position $\left(x_{0}, y_{0}\right)$, where $x_{0}$ is identified as the impact parameter, so that the starting coordinates obey the constraint

$$
x_{0}^{2}+y_{0}^{2}=\xi_{0}^{2} .
$$

Here we explore the full range of impact parameters $0 \leq x_{0} \leq \xi_{0}$. For the sake of definiteness, we consider incoming rocky planets with masses $m_{\mathrm{p}}=1$ and $10 M_{\oplus}$. If the Jovian planet has the mass and radius of Jupiter, these values correspond to dimensionless friction coefficients in the range $\alpha=1-3$. Dynamical simulations (Ketchum et al. 2011b) indicate that the expected impact velocities lie in the range $v_{0}=40-150 \mathrm{~km} \mathrm{~s}^{-1}$. This study considers a wider range of impact velocities where $v_{0}=1-10^{4} \mathrm{~km} \mathrm{~s}^{-1}$.

Examples of typical trajectories are shown in Figure 2 for a dimensionless impact parameter $x_{0}=1.5$, an initial velocity $v_{0}=40 \mathrm{~km} \mathrm{~s}^{-1}$, and rocky planet masses $m_{\mathrm{p}}=1$ and $10 M_{\oplus}$ (friction parameters $\alpha=1-3$ ). As the rocky planets spiral inward within the Jovian planets, the orbits of larger rocky planets remain relatively circular, while the orbits of smaller rocky planets become highly elliptical. In general, the bodies continuously spiral inward and approach the origin asymptotically.

There are two possible outcomes of these orbits: Either the rocky planet is captured and spirals inward, or it passes through the gaseous planet and retains enough kinetic energy 

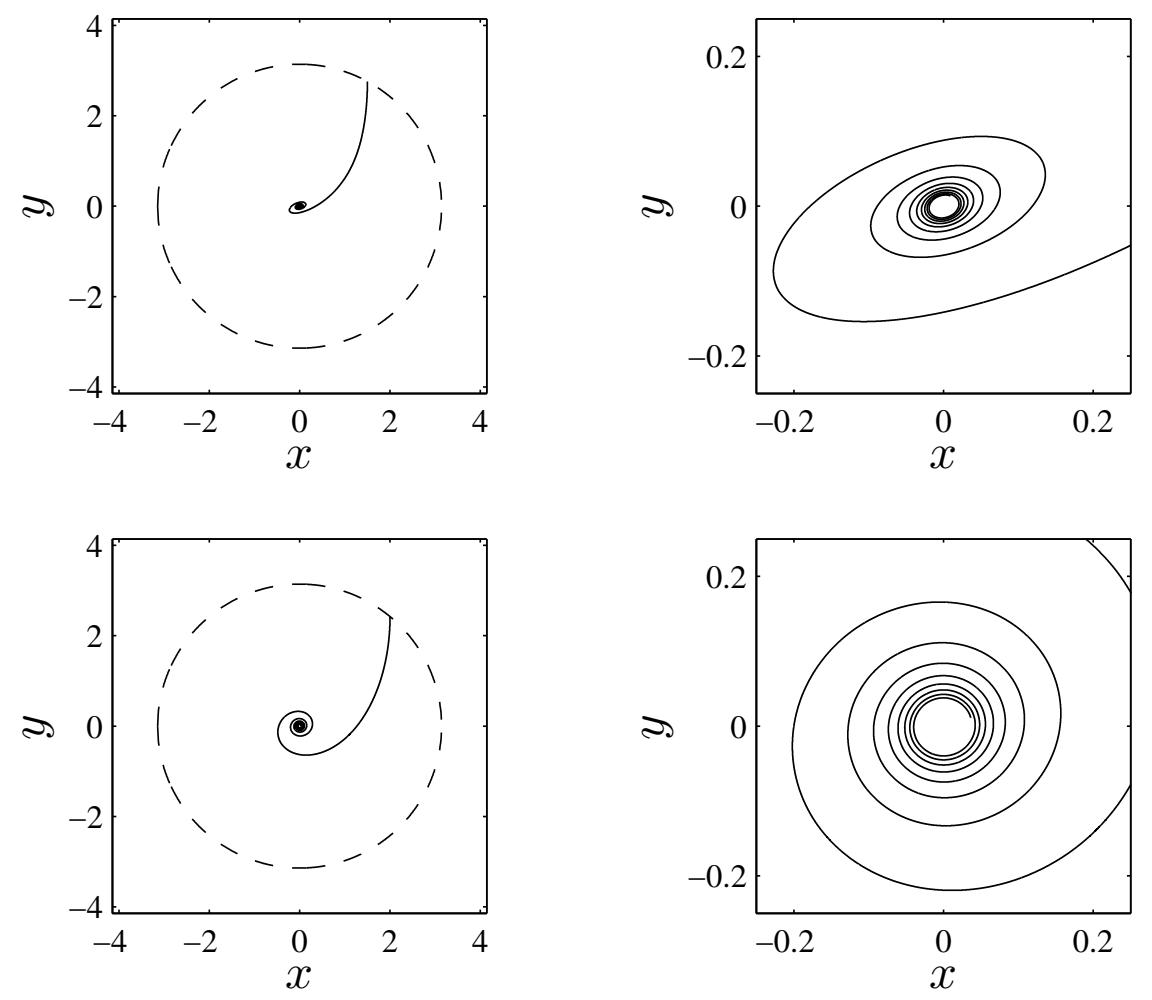

Fig. 2.- Orbits within coreless planets with polytropic index $n=1$. The top panels show results for incoming rocky planets with masses $m_{\mathrm{p}}=1 M_{\oplus}$; and bottom panels show results for $m_{\mathrm{p}}=10 M_{\oplus}$. The panels on the left show a full planetary view, whereas the panels on the right show the orbits on a smaller spatial scale near the center. Both collisions use an impact parameter $x_{0}=1.5$ and an impact velocity $v_{0}=40 \mathrm{~km} \mathrm{~s}^{-1}$. 


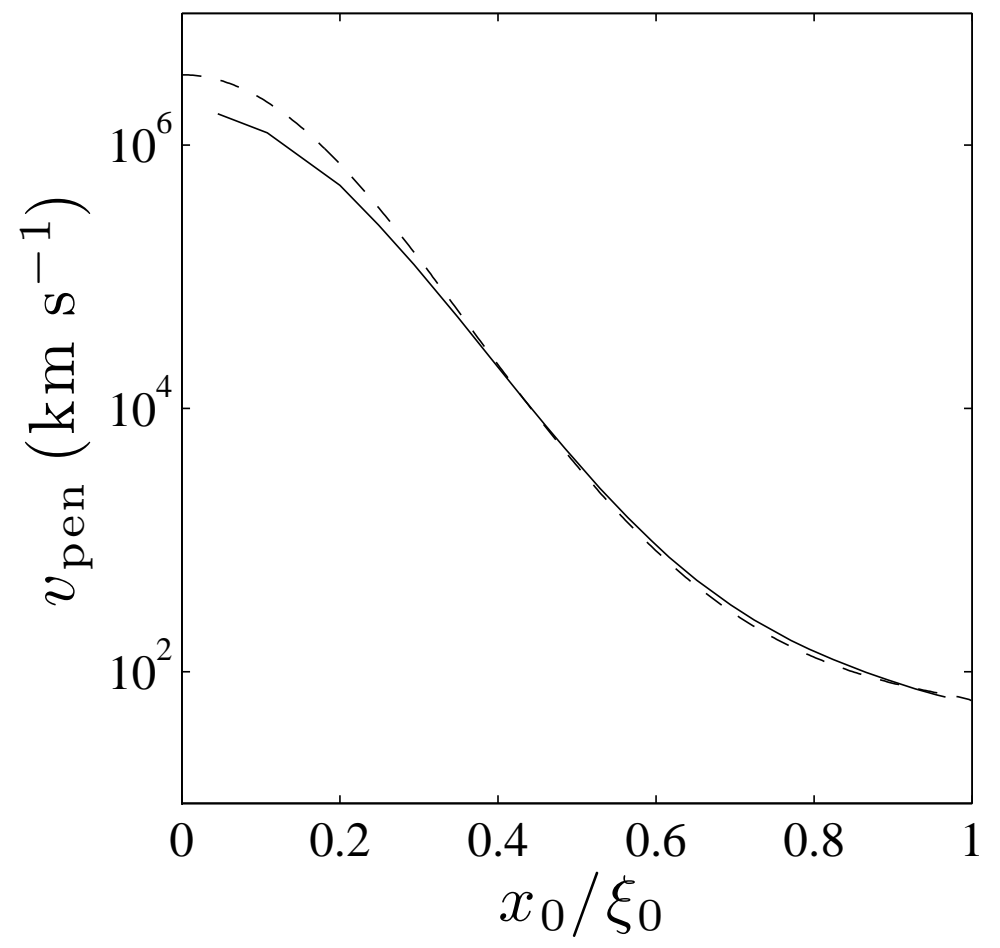

Fig. 3.- Penetration velocity as a function of impact parameter. The velocity $v_{\text {pen }}$ is the impact velocity (in $\mathrm{km} \mathrm{s}^{-1}$ ) needed for the rocky planet to pass through the gaseous giant planet, emerge from the far side, and remain uncaptured. The impact parameter is given as a fraction of the planetary radius. Here the giant planet has mass $1 M_{J}$, radius $1 R_{J}$, and $n=3 / 2$. The solid curve shows the numerical results; the dashed curve shows the analytic approximation derived in Appendix A. The approximation works well over the full range of impact parameters, with the largest discrepancy for head-on collisions $\left(x_{0}=0\right)$. 
to escape. For an incoming rocky planet with given impact parameter $x_{0}$, we can determine the initial velocity necessary for the rocky planet to avoid capture. More specifically, the critical condition for penetration requires that the rocky planet initially has enough kinetic energy so that it passes through the gaseous planet and leaves its surface traveling outward at the escape speed. This penetration speed is denoted here as $v_{\text {pen }}$ and is a function of $x_{0}$. For a given impact speed, incoming rocky planets will be captured for all impact parameters $x_{0}<x_{*}$, where the maximum impact parameter defines the effective capture cross section $\sigma\left(v_{0}\right)=\pi x_{*}^{2}$.

Here we calculate the required penetration speeds numerically using the above formulation. We also derive an analytic estimate of the penetration speed $v_{\text {pen }}$ in Appendix $\mathrm{A}$. Figure 3 shows the numerically determined penetration speeds along with the analytic approximation. The best agreement between the numerical and analytic results occurs for large impact parameters because the assumption of constant density (see Appendix $\mathrm{A}$ ) is more accurate far from the center of the planet; even for small impact parameters, however, the results agree within a factor of two. These calculations show that enormous starting velocities are required for penetration when the impact parameter is near zero, i.e., $v_{\text {pen }} \sim 10^{6} \mathrm{~km} \mathrm{~s}^{-1}$. For somewhat larger impact parameters $x_{0} \sim \xi_{0} / 2$, penetration still requires $v_{\text {pen }} \sim 1000$ $\mathrm{km} \mathrm{s}^{-1}$. As a result, the cross section for capture is large. For the expected impact speeds $v_{0}=40-150 \mathrm{~km} \mathrm{~s}^{-1}$, incoming rocky bodies will penetrate the Jovian planet only for impact parameters comparable to the radius of the planet, roughly $x_{0} \gtrsim 0.8 \xi_{0}=x_{*}$. For high-speed rocky planets with $v_{0}>100 \mathrm{~km} \mathrm{~s}^{-1}$, the effective target area (capture cross section) of the Jovian planet is thus reduced by $\sim 30 \%$.

We now consider the general case where rocky planets collide with Jovian planets containing central cores. For the sake of definiteness, we begin by choosing a standard (dimensionless) core radius $\xi_{c}=1.0$. Depending on the polytropic index, this core radius spans $\sim 20-30 \%$ of the giant planet radius, and encloses a mass of $20-30 M_{\oplus}$. Here we use three polytropic indices $(n=1,3 / 2$, and 2$)$, four terrestrial planet masses $\left(m_{\mathrm{p}}=0.1,1,10\right.$, and $20 M_{\oplus}$ ), and the full range of impact parameters $x_{0}=0-\xi_{c}$, where the initial velocities span the range $v_{0}=20-150 \mathrm{~km} \mathrm{~s}^{-1}$. The resulting trajectories, including profiles of energy dissipation and the velocities at the core, are nearly independent of the polytropic index. Given that similar results were obtained for all three choices $(n=1,3 / 2$, and 2$)$, only the results for the $n=1$ polytrope are shown here. Figure 4 shows the trajectories for rocky planets with initial speeds of $80 \mathrm{~km} \mathrm{~s}^{-1}$ and with varying impact parameter.

We note that the presence of a core has little effect on whether or not an incoming rocky planet will penetrate the Jovian planet and escape capture. As a result, the results depicted in Figure 3 remain valid - only incoming planets with large impact parameters $\left(x_{0}>0.8 \xi_{0}\right)$ 


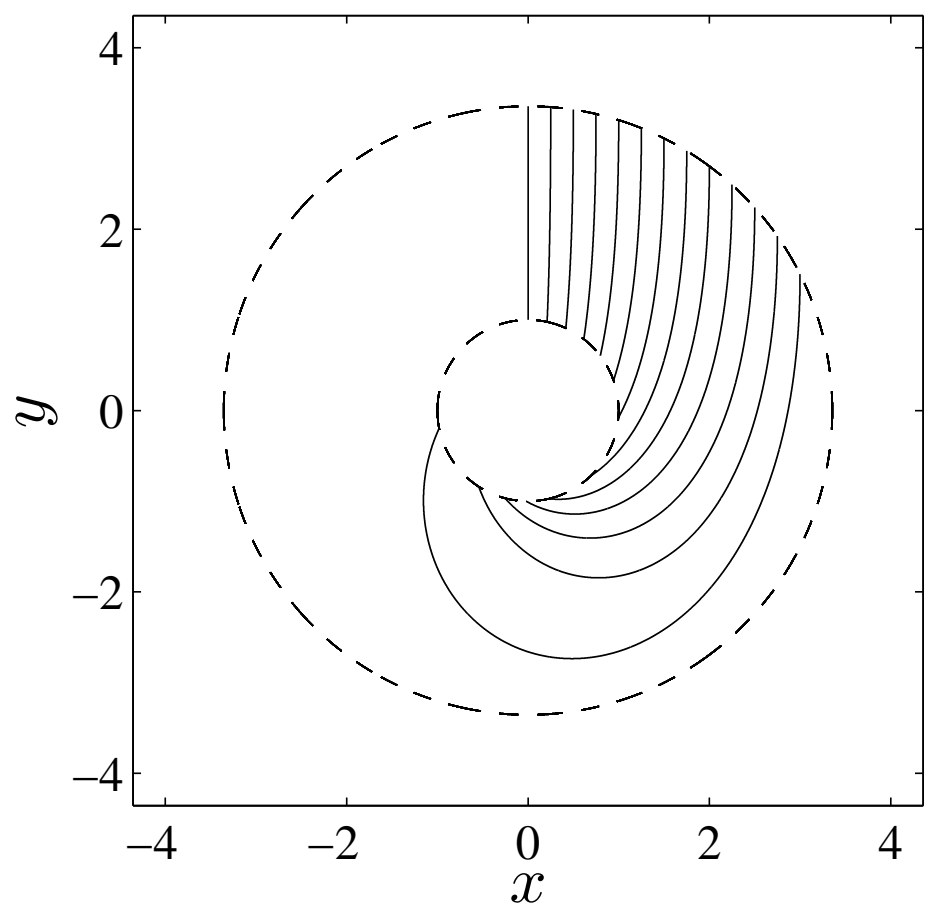

Fig. 4. - Trajectories for different impact parameters. Each curve shows the orbit of an incoming rocky body for a given impact parameter. The outer dashed circle marks the location of the outer surface of the Jovian planet; the inner dashed circle marks the location of its core, which has dimensionless radius $\xi_{c}=1$. The impact speeds, defined at the surface of the Jovian planet, are taken to be $80 \mathrm{~km} \mathrm{~s}^{-1}$. 
or incredibly large initial speeds $\left(v_{0}>1000 \mathrm{~km} \mathrm{~s}^{-1}\right)$ are able to escape.

\subsection{Energy Dissipation}

During each incoming trajectory, energy is dissipated through the action of frictional forces. Figures 5 and 6 show the total energy (kinetic and potential) of the rocky planet as a function of radial distance, measured from the center of the giant planet. These figures show results for target giant planets with the mass and radius of Jupiter, and for incoming rocky planets with $m_{\mathrm{p}}=1 M_{\oplus}$ (top panels) and $m_{\mathrm{p}}=10 M_{\oplus}$ (bottom panels). Figure 5 shows energy profiles for varying initial impact speeds, whereas Figure 6 shows energy profiles for varying impact parameters. In all cases shown, the majority of the energy is dissipated in the outer layers $\left(r / R_{P}>0.5\right)$ of the planet.

As shown in Figure 6, for sufficiently large impact parameters the energy fraction $E / E_{0}$ takes on multiple values for a given radius; on the other hand, the radius is a single-valued function of energy. This complication arises for orbits that reach an inner turning point and reverse course before finally spiraling inward (e.g., see the orbit with the largest impact parameter in Figure 4). For rocky planets with larger masses and/or giant (target) planets with lower density, the orbits display additional turning points and the energy dissipation curves show even more structure (these cases are not shown in the figures).

When the rocky planet strikes the core, its motion is arrested and any remaining kinetic energy is converted into heat. The energy remaining upon impact with the core thus determines the extent of possible core deformation as well as the amount of energy available for long-term heating. This fraction of the initial energy remaining depends on the mass of the rocky planet and the structure of the Jovian planet. Rocky planets with larger masses retain a larger fraction of their initial energy (as well as a larger absolute value of energy). For target giant planets with the properties of Jupiter (see Figures 5 and 6), $1 M_{\oplus}$ planets lose most of their initial energy, whereas $10 M_{\oplus}$ planets retain up to $30 \%$. The structure of the Jovian planet, in particular its mean density, plays the most important role. When the radius of the giant planet is doubled (which may be appropriate for young planets), the $10 M_{\oplus}$ planets can retain the majority of their initial energy (not shown). An analytic desription of the energy dissipation process is given in Appendix B.

The kinetic energy of the rocky planet at the moment of impact with the core is shown in Figure 7 as a function of both the initial velocity and impact parameter. The impact velocities vary over the range $10-75 \mathrm{~km} \mathrm{~s}^{-1}$, leading to impact energies in the range $10^{38}-10^{42}$ ergs, where these values are strongly dependent upon the rocky planet mass. 

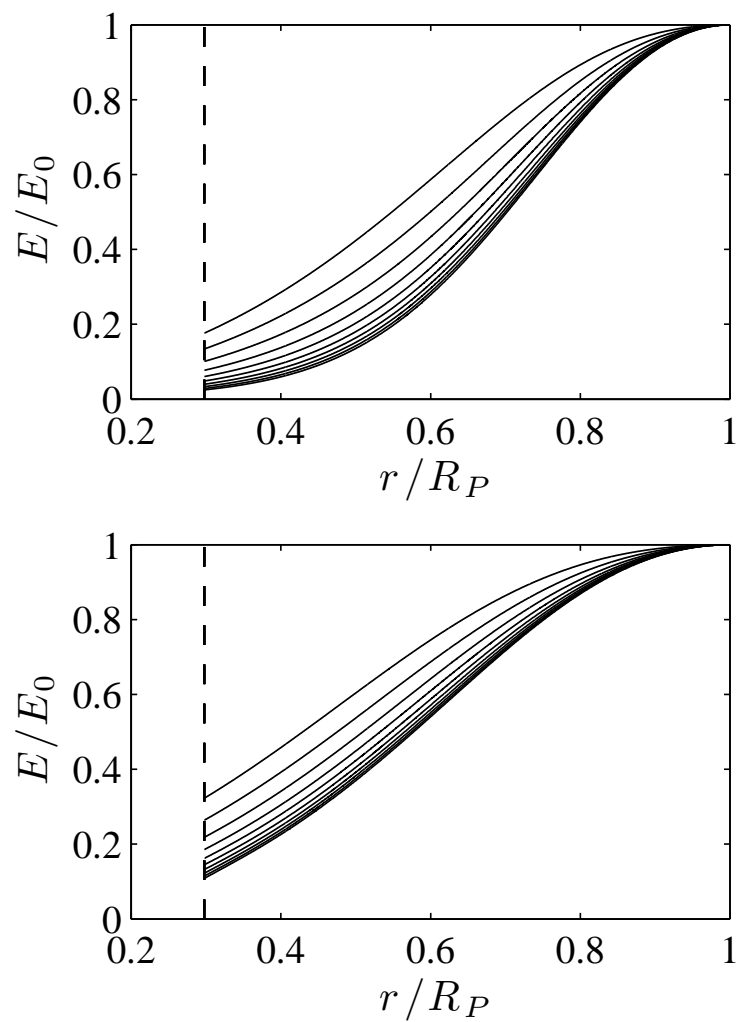

Fig. 5.- Fraction of the total energy of the rocky planet as a function of radial distance for varying initial velocities. Masses for the rocky planet are $m_{\mathrm{p}}=1 M_{\oplus}$ (top panel) and $m_{\mathrm{p}}=10 M_{\oplus}$ (bottom panel). The Jovian planet has polytropic index $n=1$, mass $M_{P}=1 M_{J}$, and radius $R_{P}=1 R_{J}$. The dashed lines indicate the core radius. 

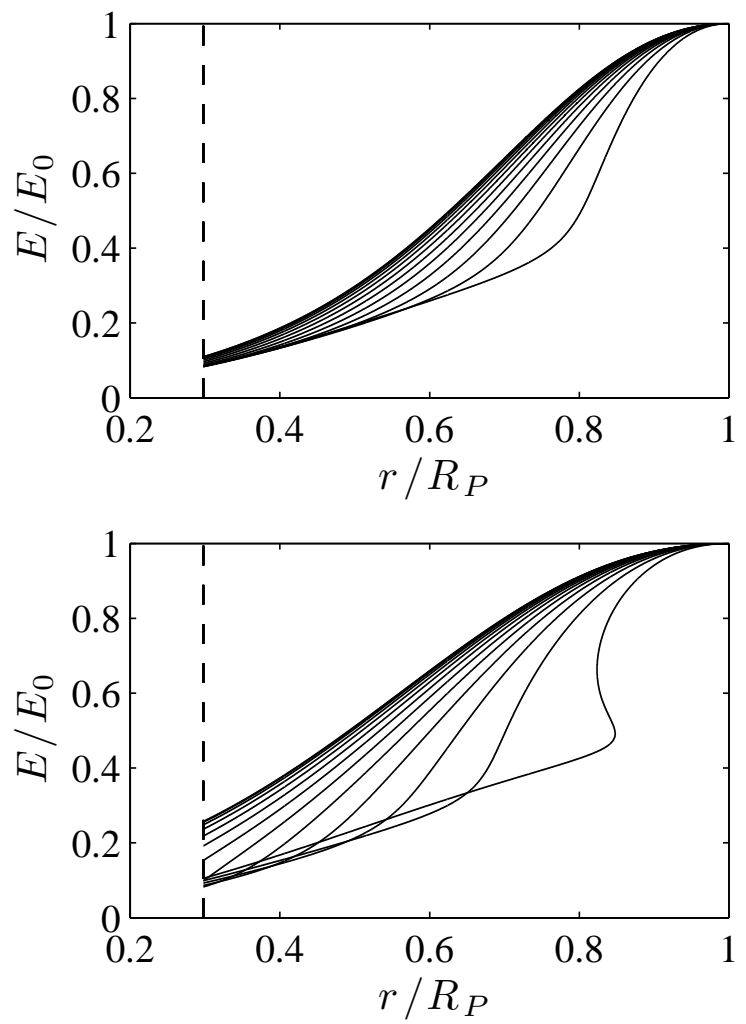

Fig. 6. - Fraction of the total energy of the rocky planet as a function of radial distance for varying impact parameters. Masses for the rocky planet are $m_{\mathrm{p}}=1 M_{\oplus}$ (top panel) and $m_{\mathrm{p}}=10 M_{\oplus}$ (bottom panel). The Jovian planet has polytropic index $n=1$, mass $M_{P}=1 M_{J}$, and radius $R_{P}=1 R_{J}$. The dashed lines indicate the core radius. 
Planets less massive than $m_{\mathrm{p}}=1 M_{\oplus}$ show little spread in their final energy (velocity) for the entire set of impact parameters and initial velocities. This result can be understood in terms of the nature of the frictional force $\left(F_{f} \sim \rho v^{2}\right)$ and the size of the friction coefficient $\alpha$. Planets with the highest velocities experience the highest levels of damping, and their speeds are quickly diminished. Furthermore, the impact parameter determines the average density encountered by the orbiting planets. Although the average density is lower for planets incident at large impact parameters, these planets must travel farther before being accreted onto the core, and are thus subjected to damping forces acting over larger distances. These effects are the most pronounced for low-mass planets, since they experience the greatest frictional forces relative to gravitational forces. Planets with masses larger than $m_{\mathrm{p}} \sim 1 M_{\oplus}$ are subjected to relatively less friction and are more sensitive to variations in the initial velocity and impact parameter. Planets incident at small impact parameters (roughly, less than the core radius) collide directly with the core and travel shorter distances. These planets thus experience less damping and retain a significant amount of energy upon impact with the core. Incoming planets with sufficiently large impact parameters do not collide directly with the core, but rather gradually spiral inward and often enter into highly elliptical orbits. The orbital path length thus increases substantially for impact parameters larger than a critical value, and the amount of energy deposited at the core decreases accordingly. For the regime of parameter space considered here, this critical impact parameter is roughly $x_{0 *} \approx 0.4 R_{P}$. Finally, we note that the curves in Figure 7 do not extend beyond $x_{0} \approx 0.9 R_{P}$; for collisions with larger impact parameter, the rocky planet does not remain bound and hence does not reach the core.

In the calculations presented so far, the mass and radius of the Jovian planet were taken to be those of Jupiter. However, many Hot Jupiters have inflated radii, with typical values $R_{P} \sim 1.2 R_{J}$ for a mass $M_{P}=1 M_{J}$. In addition, the radii of young giant planets - before they contract and cool — are even larger than those of mature planets (Burrows et al. 1997). As a result, collisions of rocky planets with inflated gaseous planets (for the same mass) must be explored. To bracket the possibilities, the previous calculations were repeated for a Jovian planet with mass $M_{P}=1 M_{J}$, but with a larger radius $R_{P}=2 R_{J}$, leading to a lower mean density of only $\rho \sim 0.1 \mathrm{~g} \mathrm{~cm}^{-3}$, ten times lower than the mean density of Jupiter. These results are shown in Figure 8. Compared to the previous case (with $R_{P}=1 R_{J}$ ), the impact energies are larger by an order of magnitude. Notice also that the curves do not extend to large impact parameters (bottom panel) because the rocky planets pass through the gaseous planet.

Another way to illustrate the effects of inflated radii for the Jovian planets is to the plot the impact energy as a function of the radius $R_{P}$ of the gas giant. These results are shown in Figure 9 for collisions with rocky planets that have starting speeds $v_{0}=50 \mathrm{~km} \mathrm{~s}^{-1}$ and 

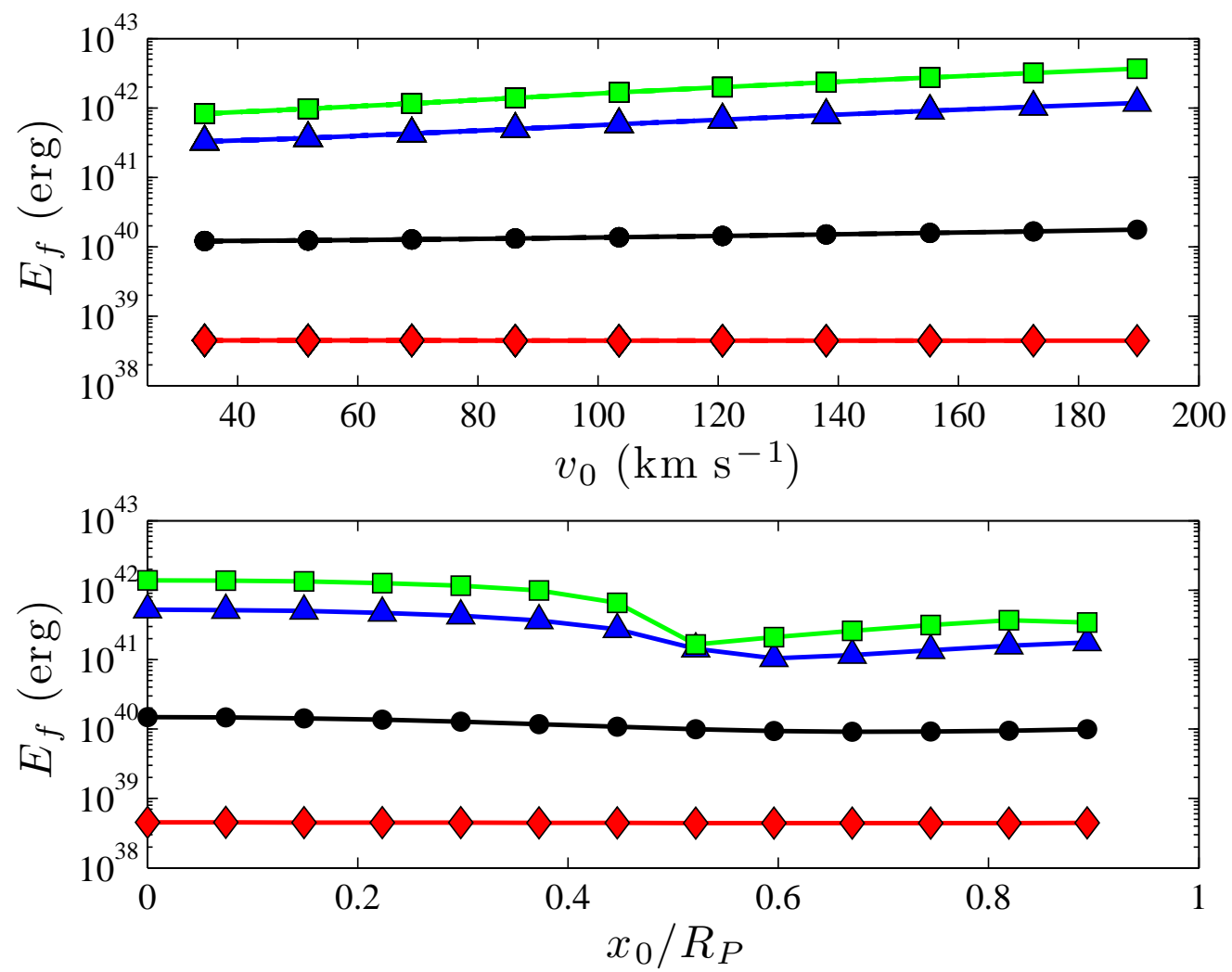

Fig. 7.- Kinetic energy of the rocky planet as it reaches the core of the Jovian planet with radius $R_{P}=R_{J}$. Impact energy is shown as a function of initial velocity for fixed $x_{0}=1$ (top panel) and as a function of impact parameter at fixed impact speed $v_{0}=80 \mathrm{~km} \mathrm{~s}^{-1}$ (bottom panel). The four curves correspond to four masses for the rocky planet, for $m_{\mathrm{p}}=$ $0.1,1,10$, and $20 M_{\oplus}$ (from bottom to top). The Jovian planet has polytropic index $n=1$, mass $M_{P}=M_{J}$, and dimensionless core radius $\xi_{c}=1$. Note that the curves do not extend beyond $x_{0} \approx 0.9$; for collisions with larger impact parameters, rocky planets pass through the gaseous planet and escape. 

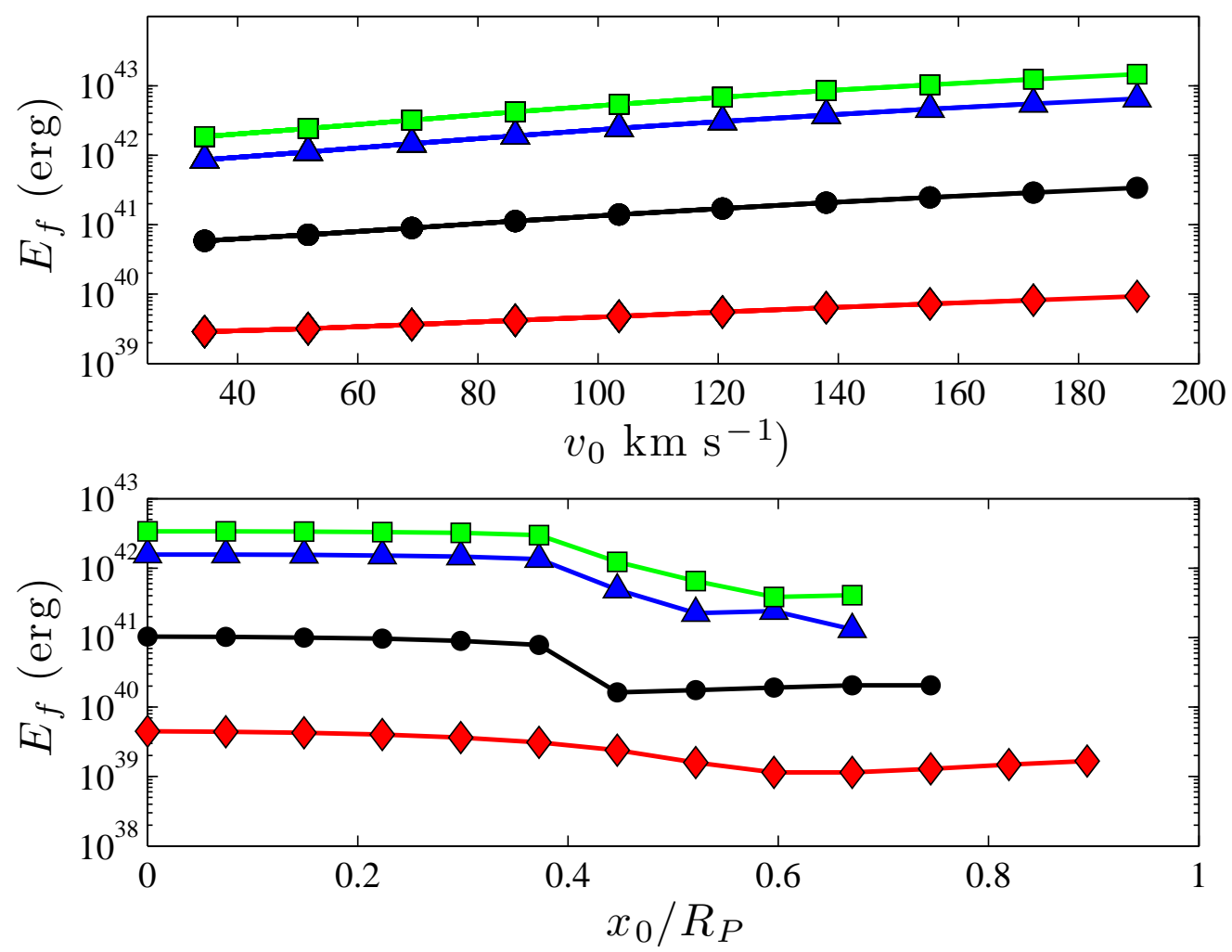

Fig. 8.- Kinetic energy of the rocky planet as it reaches the core of a young Jovian planet with large radius $R_{P}=2 R_{J}$. Impact energy is shown as a function of initial velocity for fixed $x_{0}=1$ (top panel) and as a function of impact parameter at fixed impact speed $v_{0}=$ $80 \mathrm{~km} \mathrm{~s}^{-1}$ (bottom panel). The four curves correspond to four masses for the rocky planet, for $m_{\mathrm{p}}=0.1,1,10$, and $20 M_{\oplus}$ (from bottom to top). The Jovian planet has polytropic index $n=1$, mass $M_{P}=M_{J}$, and dimensionless core radius $\xi_{c}=1$. Note that the curves do not extend all the way to $x_{0}=1$; for collisions with sufficiently large impact parameters, rocky planets pass through the gaseous planet and escape. 
varying masses $m_{\mathrm{p}}=0.1,1,10$, and $20 M_{\oplus}$. The kinetic energy $E_{f}$ remaining when the rocky planet strikes the core of the Jovian planet is a smoothly increasing function of the radius of the larger body. To leading order, the four curves shown in Figure 9 have nearly the same shape, so that they are scaled by the mass of the rocky planet. The starting energy for the $1 M_{\oplus}$ planet is $7.5 \times 10^{40} \mathrm{erg}$; for collisions with Jovian planets with $R_{P}=2.1 R_{J}$, the impact energy is about two thirds of this starting value. Rocky planets with smaller masses lose a somewhat greater fraction of their initial energy, whereas sufficiently massive rocky planets $\left(m_{\mathrm{p}} \gtrsim 10 M_{\oplus}\right)$ gain more energy by falling through the gravitational potential well of the giant planet than they lose through frictional dissipation.

This difference in the energy dissipation between Jovian planets with different radii is remarkable. The planets with inflated radii tend to dissipate far less energy in their outer envelopes, and the percentage of the initial energy remaining when the incoming rocky planets reach the core can be as high as $70 \%$. Note that this percentage represents the fraction of the rocky planet's total energy (kinetic and potential) at the surface of the Jovian planet. If we examine purely the kinetic energy, we find that the energy dissipation in the outer regions is low enough that the kinetic energy can actually increase during the inward trajectory, as it travels deeper into the potential well of the giant planet.

The consequences of these collisions differ greatly for young Jovian planets with low densities and mature planets with higher densities. Mature planets are able to dissipate much more of the incoming energy near the surface, where heat is transported efficiently by radiation. In such cases, the energy of the impact is expected to radiate away quickly, and the structure of the giant planet should recover on short time scales. On the other hand, young planets with large radii tend to dissipate little energy in the radiative zones, and the majority of the impact energy is delivered to the core, where it becomes trapped and is available for long-term heating. The evolutionary stage of the Jovian planet is therefore an important factor in determining the long-term effects of these collisions. Young inflated planets will be far more susceptible to core enhancement and prolonged heating than mature planets. Similarly, Hot Jupiters will be more susceptible than giant planets farther from their stars.

\section{Tidal Disruption}

For purposes of finding their orbits, the impinging rocky planets have thus far been treated as indestructible point particles. In this section we consider the possibility that these planets can be disrupted by tidal forces. If the incoming planet is destroyed, several outcomes are possible. Depending on the interior conditions of the giant planet, the rocky 


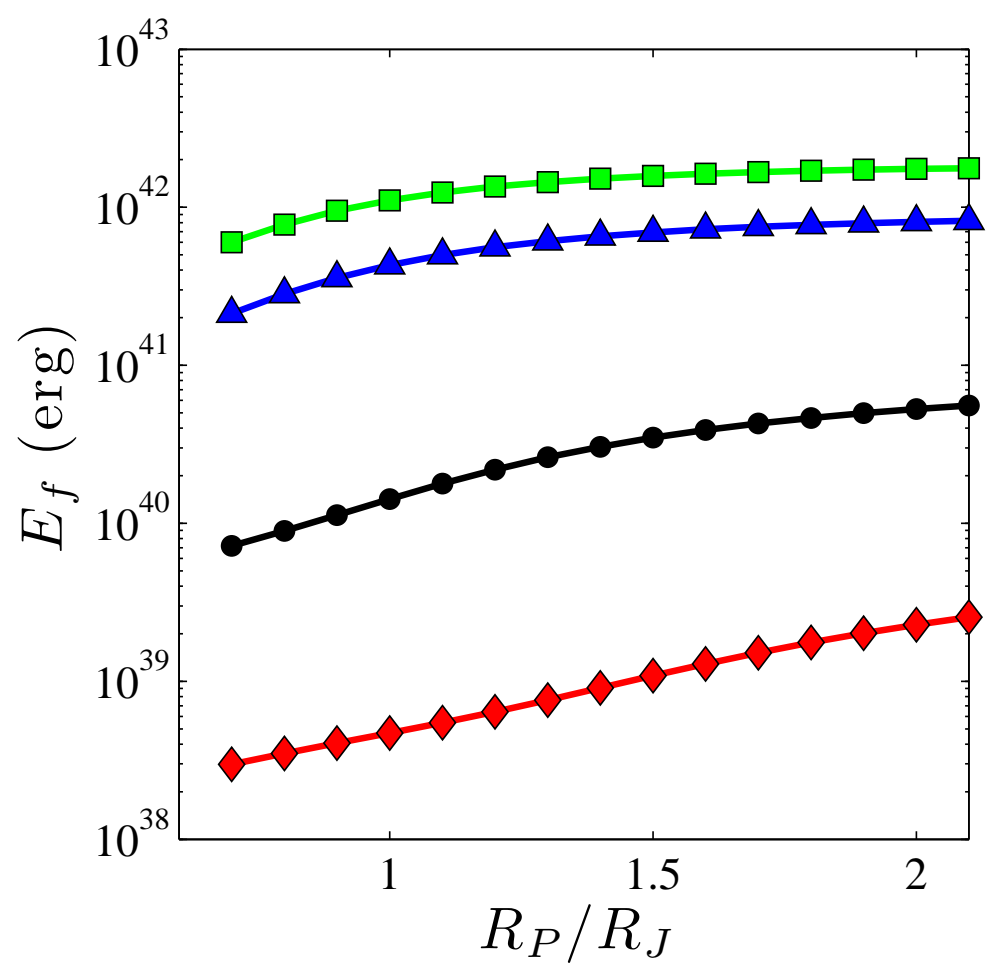

Fig. 9.- Effects of the Jovian planet radius on collisions with rocky planets. Kinetic energy of the rocky planet as it reaches the core of the Jovian planet is shown as a function of Jovian planetary radius $R_{P}$. The four curves correspond to rocky planet masses $m_{\mathrm{p}}=0.1$, 1, 10, and $20 M_{\oplus}$ (from bottom to top). The Jovian planet has polytropic index $n=1$, mass $M_{P}=M_{J}$, and dimensionless core radius $\xi_{c}=1$. For all cases shown, the initial speed (evaluated at the surface of the Jovian planet) is $v_{0}=50 \mathrm{~km} \mathrm{~s}^{-1}$ and the impact parameter $x_{0}=0$ (head-on collisions). 
planet remnants could either become accreted onto the core, or uniformly enrich the planetary interior with heavy elements. A full determination of the outcome is computationally expensive and is beyond the scope of this present work; in addition, it requires a greater understanding of Jovian planet interiors (e.g., including convection) than is currently available. In this section we consider how tidal forces act on the incoming rocky planets and determine the likelihood of these bodies reaching the core (of the Jovian planet) relatively intact. These results allow us to estimate the maximum amount of mass and energy deposited at the core.

At any point in the trajectory, the tidal force (per unit mass) exerted on the rocky planet can be approximated by the difference in the gravitational acceleration at the near and far surfaces,

$$
\Delta F=\frac{G M(r)}{r^{2}}-\frac{G M(r+\Delta r)}{(r+\Delta r)^{2}},
$$

where $\Delta r=2 r_{\mathrm{p}}$ (the rocky planet diameter). Note that the tidal forces arise due to the difference in the enclosed mass as well as the difference in the radial distance. Since the tidal forces are not spherically symmetric with respect to the rocky planet, this treatment introduces an approximation. Nonetheless, if the rocky planet is to withstand tidal forces, the tidal acceleration given by equation (22) must be less than the surface gravity of the rocky planet. Equating these quantities yields the critical condition for survival, i.e.,

$$
\left|\frac{G M(r)}{r^{2}}-\frac{G M(r+\Delta r)}{(r+\Delta r)^{2}}\right|=\frac{G m_{\mathrm{p}}}{r_{\mathrm{p}}^{2}} .
$$

This expression can be simplified by expanding in $(\Delta r) / r \ll r$ and keeping only the leading order terms. With these approximations, the expression (22) for the tidal force takes the more familiar form

$$
\Delta F=\frac{4 r_{\mathrm{p}} G M(r)}{r^{3}}-8 \pi r_{\mathrm{p}} G \rho(r) .
$$

Near the surface of the Jovian planet, the density is small enough so that the second term on the right hand side is small compared to the first. After dividing by $r_{\mathrm{p}}$, we obtain a rough criterion for the survival of the rocky planet,

$$
\frac{4 M(r)}{r^{3}} \leq \frac{m_{\mathrm{p}}}{r_{\mathrm{p}}{ }^{3}}
$$

In other words, the incoming body will be tidally disrupted if its mean density is less than (about) four times the mean density of the giant planet. Note that equation (25) is valid near the surface of the Jovian planet, where the differential change in mass and the ratio $(\Delta r) / r$ are relatively small. Here we consider rocky planets with radii $r_{\mathrm{p}} \sim 1 R_{\oplus}$, and giant planets with radii $R_{P} \sim 10 R_{\oplus}$, so that $(\Delta r) / r \approx 0.2$ at the surface and increases with decreasing radial position. As a result, the approximation scheme deteriorates as the rocky 
planet moves inward. Here we use equation (25) to provide an order of magnitude estimate for the tidal stretching at the Hot Jupiter surface, but use the full form of equation (22) to assess the prospects for rocky planet survival (see below).

Notice that the two sides of equation (25) are nearly equal for a pair of planets with the mean densities of Jupiter and Earth (where $\langle\rho\rangle \sim 1.3 \mathrm{~g} \mathrm{~cm}^{-3}$ and $\sim 5.5 \mathrm{~g} \mathrm{~cm}^{-3}$, respectively). As a result, an Earth-like planet can be tidally disrupted by a giant planet like our Jupiter, but very well may survive if the radius of the Jovian planet is somewhat larger (so that its density is lower). Since both Hot Jupiters and young Jupiters (in any orbit) have inflated radii, incoming rocky planets are likely to survive tidal disruption, reach their centers, and increase the masses of their cores.

The ratio of the tidal force to the surface gravity of the rocky planet can be calculated as a function of radial distance using equation (23). Here we explore a variety of planetary properties. The Jovian planet mass was held constant at $M_{P}=1 M_{J}$ and the radius varied over the range $R_{P}=1-2 R_{J}$, leading to average densities $\langle\rho\rangle \sim 0.1-1.0 \mathrm{~g} \mathrm{~cm}^{-3}$. The results are shown in Figure 10 for $R_{P}=1 R_{J}$ and in Figure 11 for $R_{P}=1.2 R_{J}$, where each curve represents a different rocky planet density. The latter value corresponds to a typical radius for observed Hot Jupiters, which are inflated relative to expectations (e.g., Laughlin et al. 2011). For even larger values of $R_{P}$ (not shown), the densities of the Jovian planet are small enough that little tidal disruption takes place. The expected range of (mean) densities for rocky planets is $\rho \approx 2-10 \mathrm{~g} \mathrm{~cm}^{-3}$ (Valencia et al. 2007), with planet masses $m_{\mathrm{p}}=0.1-20$ $M_{\oplus}$. Note that planets less massive than $\sim 1 M_{\oplus}$ are not expected to survive. Most of the calculations were performed for coreless polytropes of index $n=1$; adding a core does not significantly alter the strength of the tidal forces (and hence these curves).

Notice that the tidal force $\Delta F$ vanishes at radii $r \sim R_{P} / 2$. The tidal force becomes negative for smaller radii, indicating that an orbiting planet will be first be stretched and subsequently compressed during its inward trajectory. In the center of the Jovian planet (where $r \rightarrow 0$ ), the compression reaches a maximum and the force given by equation (24) formally approaches a well-defined limit

$$
\lim _{r \rightarrow 0} \Delta F=-\frac{8 \pi}{3} G \rho_{c} r_{\mathrm{p}}
$$

Note that this is a (mathematically) formal result, because we are taking the limit $r \rightarrow 0$ but the rocky planet has a finite size $r_{\mathrm{p}}$.

The area between the dashed lines (set where $(\Delta F) / f_{\mathrm{p}}= \pm 1$ ) in Figures 10 and 11 indicates the regions where the surface gravity of the rocky planet can withstand the tidal acceleration. If the ratio $(\Delta F) / f_{\mathrm{p}}>1$, the outer layers of the rocky planet are stripped away; if $(\Delta F) / f_{\mathrm{p}}<-1$, the planet experiences devastating levels of compression. Here we 

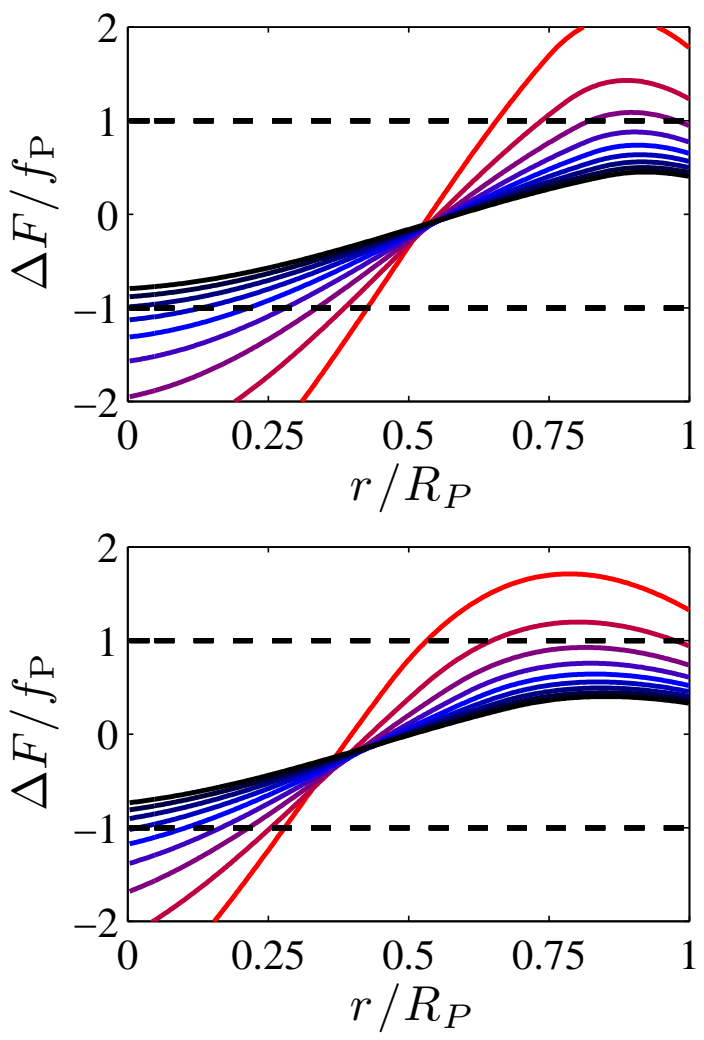

Fig. 10.- Ratio of tidal forces to rocky planet surface gravity $f_{\mathrm{p}}$ as a function of radial distance (where $f_{\mathrm{p}}=G m_{\mathrm{p}} / r_{\mathrm{p}}{ }^{2}$ ). The Jovian planet is taken to have no core, mass $M_{P}=M_{J}$, radius $R_{P}=R_{J}$, and $n=1$. Results are shown for rocky planets with mass $m_{\mathrm{p}}=1 M_{\oplus}$ (top panel) and $m_{\mathrm{p}}=10 M_{\oplus}$ (bottom panel). In each panel, the curves correspond to different mean densities of the rocky planet, ranging from $\rho_{\mathrm{p}}=2 \mathrm{~g} \mathrm{~cm}^{-3}$ (top red curve) to $\rho_{\mathrm{p}}=10 \mathrm{~g}$ $\mathrm{cm}^{-3}$ (bottom black curve). The region between the dashed lines indicates radial locations where the rocky planet can survive. 

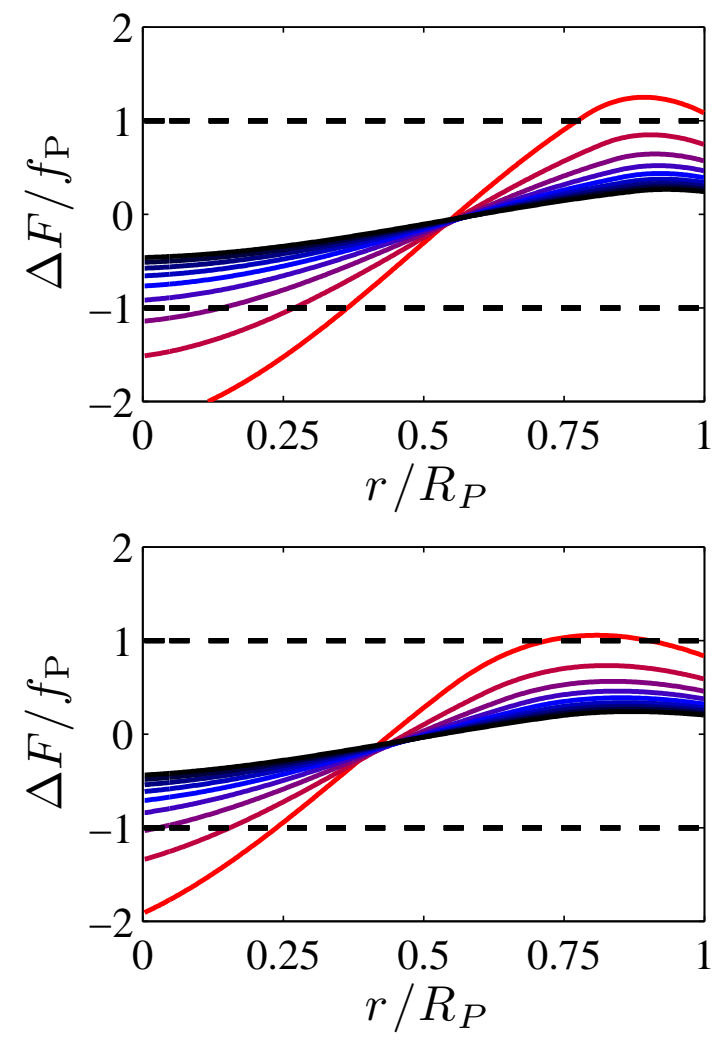

Fig. 11. - Ratio of tidal forces to rocky planet surface gravity as a function of radial distance for an inflated gaseous planet. Here the Jovian planet is taken to have no core, mass $M_{P}=M_{J}$, radius $R_{P}=1.2 R_{J}$, and $n=1$. Results are shown for rocky planets with mass $m_{\mathrm{p}}=1 M_{\oplus}$ (top panel) and $m_{\mathrm{p}}=10 M_{\oplus}$ (bottom panel). In each panel, the curves correspond to different mean densities of the rocky planet, ranging from $\rho_{\mathrm{p}}=2 \mathrm{~g} \mathrm{~cm}^{-3}$ (top red curve) to $\rho_{\mathrm{p}}=10 \mathrm{~g} \mathrm{~cm}^{-3}$ (bottom black curve). The region between the dashed lines indicates radial locations where the rocky planet can survive. 
consider a rocky planet to survive whenever the condition $\left|(\Delta F) / f_{\mathrm{p}}\right|<1$ is satisfied all the way to the core. All but the smallest planets are able to penetrate deep into the interior (at least $50-70 \%$ of the distance to the center) before experiencing severe tidal disruption; in many cases, the rocky planet reaches the core intact. As expected, the small planets $\left(m_{\mathrm{p}} \sim 0.1 M_{\oplus}\right)$ must be very dense to survive the entire trajectory, and we exclude them in the remaining discussion. Here we focus on rocky planets with masses $m_{\mathrm{p}}=1,10$, and $20 M_{\oplus}$. For Jovian planets with the mean density of Jupiter $\left(1.2 \mathrm{~g} \mathrm{~cm}^{-3}\right)$, a rocky planet with $m_{\mathrm{p}}=1 M_{\oplus}$ must have density greater than $\rho_{\mathrm{p}} \approx 5-6 \mathrm{~g} \mathrm{~cm}^{-3}$ for survival; larger rocky planets with $m_{\mathrm{p}}=10 M_{\oplus}$ can survive with somewhat lower density $\rho_{\mathrm{p}} \approx 3-4 \mathrm{~g} \mathrm{~cm}^{-3}$. On the other hand, for Jovian planets with sufficiently high densities (roughly, where the inequality of equation [25] is violated), the tidal forces at the surface can exceed the rocky planet surface gravity, and such planets can be tidally disrupted before even entering the atmosphere.

After a rocky planet is tidally disrupted, its fate remain unclear. The resulting rocky debris is unlikely to continue the journey toward the core, because smaller objects are more susceptible to frictional dissipation (and further tidal disruption). The rocky debris is likely to remain in the outer envelope of the giant planet and enrich the atmosphere, but its longer-term fate should be the subject of further study. The outcome will depend on, among other things, the opacity of the Jovian planet. For example, high opacities lead to convection, which could cause the rocky debris to be uniformly mixed into the gaseous planetary interior.

In summary, we find that large rocky planets $\left(m_{\mathrm{p}} \sim 10 M_{\oplus}\right)$ have a good chance of reaching the core intact; even planets with $m_{\mathrm{p}} \sim 1 M_{\oplus}$ are able to survive in many cases, as long as the Jovian planet density is sufficiently low. As a result, these collisions provide a viable mechanism for allowing significant amounts of rocky material to be be accreted onto the cores of gaseous planets, especially those with inflated radii like young Jovian planets and much of the observed Hot Jupiter sample.

\section{Conclusion}

This paper explores collisions between Jovian planets and smaller terrestrial bodies in order to identify possible changes in the structure of the larger planets. This work focuses on the scenario where the Jovian planet has migrated inward and entered into a tight orbit, and subsequently experiences collisions with rocky planets that migrate later. However, many of the results apply to other parts of parameter space, e.g., the possible accretion of cores by gaseous planets formed via gravitational instability (e.g., Boss 1997; Boley 2009; Boley et al. 2011; Helled \& Shubert 2009). These collisions can affect the structure of the target giant 
planets in three ways: [a] The metallicity is increased, [b] The core size can be increased, and $[\mathrm{c}]$ A substantial amount of energy is imparted to the giant planet. For Hot Jupiters, these impacts could thus help explain the observed anomalous radius distribution.

Rocky planets are efficiently captured by giant planets and thereby increase the metallicity of the larger body. For sufficiently high impact speeds $v_{0}$ and large impact parameters $x_{0}>x_{*}$, the projectile planet can pass through the giant planet and emerge out the other side. For nearly head-on collisions, the initial velocity required to to escape capture is much higher $\left(v_{\text {pen }} \sim 10^{4} \mathrm{~km} \mathrm{~s}^{-1}\right)$ than the expected impact speeds $v_{0} \approx 40-150 \mathrm{~km} \mathrm{~s}^{-1}$ (Ketchum et al. 2011b). With these latter speeds, incoming planets can escape only for large impact parameters $x_{0} \gtrsim 0.8 R_{P}$, where the densities are low. In other words, penetration is possible when the incoming rocky planet skims the surface of the Jovian target. As a result, most rocky planets lose enough kinetic energy through their initial passage that they remain gravitationally bound and are captured (see Figure 3, an analytic estimate of the required penetration speeds is given in Appendix (A). Nonetheless, the capture cross section of the giant planet can be reduced; for typical collision speeds $v_{0} \sim 100 \mathrm{~km} \mathrm{~s}^{-1}$, the cross section is smaller than the geometrical area by $\sim 30 \%$.

These collisions provide a plausible mechanism for explaining the massive cores that have been inferred for observed Hot Jupiters. Although metallicity increases are essentially automatic through such collisions, core masses can only increase if the rocky planets avoid tidal disruption and survive to reach the central regions. The expected parameter space includes cases where the impinging planets survive and cases where they are completely disrupted. As a benchmark, rocky planets with the mass and radius of Earth live near the threshold for tidal destruction when they collide with giant planets having the mass and radius of Jupiter. However, sufficiently dense projectile planets or inflated giant (target) planets allow for the rocky planets to survive all the way to the central regions (see Figures 10 and 11). This finding is relevant because such collisions are expected to take place early in the system's evolution when the giant planets are larger, and also because Hot Jupiters are subject to additional heating sources and are thus larger in radius for a given mass. As noted above, this process also provides a mechanism through which planets formed via gravitational instability can acquire cores.

For collisions to significantly alter the metallicity or the core mass of a giant planet, it must accrete rocky bodies that represent total masses $\Delta M \sim 10-100 M_{\oplus}$. The accumulation of such mass increments could take place through collisions with many smaller rocky bodies or via fewer larger bodies. Large rocky bodies, with mass $m_{\mathrm{p}}>10 M_{\oplus}$, are more effective for several reasons: First, larger rocky bodies are subject to less eccentricity damping during migration, and are thus more likely to collide with the giant planets. More specifically, 
eccentricity damping will be small enough to allow collisions when the rocky planets are large enough to partially clear gaps (Artymowicz 1993; Klev et al. 2004), which occurs when their Hill sphere exceeds the disk scale height (Crida et al. 2008; Papaloizou \& Terquem 2006). These considerations indicate that relatively large rocky planets (with mass $m_{\mathrm{p}}$ $\approx 10-20 M_{\oplus}$ ) are favored in order to achieve partial gap-clearing, reduced eccentricity damping, and ultimately collisions. In addition, collisions with larger rocky bodies allow for the required mass increments to be realized through a lower number of encounters. Finally, larger rocky bodies are more likely to survive tidal disruption and reach the central regions (see Figures 10 and 11). On the other hand, if the circumstellar disk has high levels of turbulence, small rocky planets are subject to stochastic migration (e.g., Adams \& Bloch 2009), and can still experience encounters with gas giants. Such encounters often result in collisions (Ketchum et al. 2011b) when they take place at small semimajor axes $(a \lesssim 0.1$ $\mathrm{AU}$ ); for larger $a \gtrsim 1 \mathrm{AU}$, most encounters result in ejection of the smaller rocky planet, with collisions taking place only $\sim 10 \%$ of the time (Ketchum et al. 2011a).

Collisions provide a substantial amount of energy to the target giant planet. For rocky planets with mass $m_{\mathrm{p}}=10 M_{\oplus}$ and initial speed $v_{0}=60 \mathrm{~km} \mathrm{~s}^{-1}$, the kinetic energy transferred to the giant planet is $\Delta E \sim 10^{42} \mathrm{erg}$. If, for example, this energy is radiated over a time scale of $1 \mathrm{Gyr}$, the associated increment in planetary luminosity would be $\Delta L_{P} \approx 4 \times 10^{18}$ $\mathrm{W}$, which is large enough to affect the internal structure of the planet (Bodenheimer et al. 2003). However, in order for these collisions to provide an effective (long-term) heating source, most of the energy must be deposited deep in the Hot Jupiter interior, in regions of high opacity. For a given rocky planet mass, the amount of energy deposited depends on the density of the Jovian planet. For a Jovian planet with the mass and radius of Jupiter, most of the kinetic energy is dissipated in the outer layers of the planet. At the other end of the possible range, for a Jovian planet with the mass of Jupiter and an inflated radius $R_{P}=2 R_{J}$, little energy is dissipated in the outer layers and a sizable fraction remains upon impact with the core (see Figures [5] and 6 , and Appendix [B).

An important unresolved issue is the question of how quickly the collision-induced energy is transferred out of the planet. Collisions with large terrestrial planets deposit enough energy in the central regions of the Jovian planets to potentially affect their structure. This energy could even play a role in explaining the radius anomalies of the transiting exoplanets if the time scales for energy loss are sufficiently long. In future work, interior models of Hot Jupiters should be modified to include this possible heat source.

As shown herein, incoming rocky bodies are sometimes tidally destroyed after entering the surface of the giant planet (see also Anic et al. (2007) and Li et al. (2010) for detailed simulations of specific cases). This work indicates that most planets heavier than $m_{\mathrm{p}} \sim 10 M_{\oplus}$ 
are able to withstand tidal disruption (see Figures 10 and 11). However, this work focuses on frictional forces and tidal disruption, but does not consider the possible destruction of incoming rocky planets through heating and ablation. Through both ablation and tides incoming planets continuously lose mass throughout their descent, and the relative importance these effects should be studied in future work. If ablation is strong enough, the rocky planets could completely disintegrate before reaching the core, even if they can survive tidal disruption. Ablation will thus increase the overall metallicity of the Jovian planet atmosphere and reduce the amount of kinetic energy and mass delivered to the core. Since the calculations of this paper do not include ablation, the quantities of heat and heavy elements deposited deep in the Jovian planet interior could be lower than estimated herein.

Although ablation effects are important for smaller rocky bodies such as planetesimals (Benvenuto \& Brunini 2008), they are not expected to play a dominant role for full-sized planets with masses $m_{\mathrm{p}}=1-10 M_{\oplus}$ (Anic et al. 2007; Li et al. 2010). In rough terms, an incoming projectile planet is expected to be destroyed only after it encounters a gas mass larger than its own mass (Korycansky \& Zahnle 2005). The mass in gas $\Delta m$ encountered on an inward trajectory is roughly given by $\Delta m=\Sigma_{\mathrm{p}} \rho_{P} R_{P}$, so that survival requires a minimum size $r_{\mathrm{p}} \gtrsim 3 \rho_{P} R_{P} /\left(4 \rho_{\mathrm{p}}\right) \sim 1-2 R_{\oplus}$. As a result, the larger rocky planets of interest here are likely to survive ablation, but smaller bodies are susceptible to destruction.

The results of this paper show that the mean density of the giant planet plays a defining role in determining the consequences of these collisions. The average density helps determine whether or not incoming rocky planets can survive tidal disruption and affects the radial layers where kinetic energy is deposited. Since giant planets are born with enlarged radii, this density dependence translates into a dependence on the age of the planet. A rocky planet that collides with a young giant planet (with low density) can have dramatically different consequences than a rocky planet that collides with a mature giant planet (with higher density). The environment in which the giant planet resides plays an additional role. Since Hot Jupiters often remain significantly inflated throughout their evolution, this subset of extrasolar planets should be even more sensitive to these collisions. This lends promising support for the proposal that planetary impacts could help explain, in part, the anomalous radius distribution of the observed Hot Jupiters.

This paper resulted from the senior thesis of KA and benefited from discussions with many colleagues, including Tony Bloch, Jake Ketchum, and Greg Laughlin. This work was supported by NASA grant NNX11AK87G from the Origins of Solar Systems Program, and by NSF grant DMS-0806756 from the Division of Applied Mathematics. 


\section{A. Analytic Estimate for the Penetration Velocity}

In this Appendix, we derive an analytic estimate for the penetration velocity $v_{\text {pen }}$, the speed required for an impinging rocky planet to pass through a Hot Jupiter and thereby avoid capture. As shown below, the required velocity is much larger than the gravitational escape speed $v_{\text {esc }}$ from the planet. As a result, gravity can be neglected to leading order, and the equation of motion simplifies to the form

$$
\frac{d \mathbf{v}}{d \tau}=-\alpha f^{n} v^{2} \hat{v}=-\alpha f^{n} v(\dot{x} \hat{x}+\dot{y} \hat{y})
$$

where all of the variables are dimensionless (defined in the text). By definition, the initial velocity lies in the $\hat{y}$ direction and the initial spatial position is given by $y_{0}=-L$, where $L>0$. Further, since the required speeds are large, the path of the rocky planet through the Hot Jupiter can be approximated as a straight line (for cases of interest where the rocky planet is not captured). As a result, we can set $\dot{x} \approx 0$ and $v=\dot{y}$. With these simplifications, equation (A1) becomes

$$
\frac{d v}{d \tau}=-\beta v^{2}, \quad \beta \equiv \alpha\left\langle f^{n}\right\rangle
$$

where we have made the approximation $f^{n}=\left\langle f^{n}\right\rangle$. This differential equation is easily integrated. Applying the boundary condition $v=v_{0}$ at time $\tau=0$, we obtain the result

$$
v=\frac{d y}{d \tau}=\frac{v_{0}}{1+\beta \tau v_{0}}
$$

Integrating equation (A3) for $y(\tau)$ yields the solution

$$
y-y_{0}=\beta^{-1} \ln \left(1+v_{0} \beta \tau\right) .
$$

We can combine equation( $(\mathrm{A3})$ and (A4) to find the speed $v$ as a function of position $y$,

$$
v=v_{0} \mathrm{e}^{-\beta\left(y-y_{0}\right)}
$$

In order for the rocky planet to penetrate the Jovian planet, the speed must exceed the escape speed $\left(v>v_{\text {esc }}\right)$ at the location $y=+L=-y_{0}$. The minimum initial speed $v_{\text {pen }}$ required for penetration is thus given by the condition

$$
v_{\text {esc }}=v_{\text {pen }} \mathrm{e}^{-2 \beta\left|y_{0}\right|}, \quad \text { or } \quad v_{\text {pen }}=v_{\text {esc }} \mathrm{e}^{2 \beta\left|y_{0}\right|} .
$$

Note that the escape speed is given by $v_{\mathrm{esc}}=\left(2 \mu_{0} / \xi_{0}\right)^{1 / 2}$ in terms of dimensionless variables.

Next we find the dependence of the penetration speed $v_{\text {pen }}$ on the impact parameter $x_{0}$. Here we estimate the average density by approximating the density profile of the Jovian 
planet as a Gaussian function, where the peak is a function of the impact parameter. For head on collisions, with $x_{0}=0$, we assume $\left\langle f^{n}\right\rangle \approx 1 / 2$. The constant $\beta$ is thus given by

$$
\beta=\frac{1}{2} \alpha \exp \left[-\gamma x_{0}^{2}\right]
$$

where we have introduced an unspecified parameter $\gamma$, which is expected to be of order unity. We find that reasonable agreement with numerical results can be obtained using $\gamma \approx 1 / 4$ (see Figure 31). Equations (A6) and (A7) together yield a simple relation between the initial velocity and the impact parameter. In the limiting case of grazing collisions, where $x_{0}=\xi_{0}$ and $y_{0}=0$, the penetration speed is equal to the escape velocity $v_{\text {pen }}=v_{\text {esc }}$. On the other hand, for head-on collisions where $x_{0}=0$ and $y_{0}=-\xi_{0}$, the penetration speed attains its maximum value $v_{\text {pen }} \approx v_{\text {esc }} \exp \left[\alpha \xi_{0}\right]$. Note that even though the parameters $\alpha$ and $\xi_{0}$ are "of order unity", their typical values are $\sim 3$, so that the exponential factor is large and $v_{\text {pen }} \gg v_{\text {esc }}$.

The above derivation assumes that $\left\langle f^{n}\right\rangle \approx 1 / 2$. For the particular case of an $n=1$ polytrope with no central core $\left(\xi_{c} \rightarrow 0\right)$, we can directly test this approximation:

$$
\left\langle f^{n}\right\rangle=\langle f\rangle=\frac{1}{\pi} \int_{0}^{\pi} \frac{\sin \xi}{\xi} d \xi=\frac{1}{\pi} \operatorname{Si}(\pi),
$$

where $\operatorname{Si}(x)$ is the sine integral (Abramowitz \& Stegun 1970), which has the known value $\operatorname{Si}(\pi) \approx 1.85194$, so that $\left\langle f^{n}\right\rangle \approx 0.5895$. Thus, the approximation is reasonable.

\section{B. Analytic Estimate for Energy Dissipation}

In this Appendix, we provide an analytic estimate for the energy $E_{f}$ remaining when an incoming rocky planet reaches the core of the Jovian planet. The incoming planet has initial kinetic energy $E_{0}=v_{0}^{2} / 2$, where the speed $v_{0}$ is evaluated when the rocky planet reaches the surface of the Jovian planet. For the particular case of direct collisions, with zero impact parameter, the incoming planets travel along a radial path from the gas giant surface to the core. The amount of energy $W$ dissipated along this path is given by

$$
W=\alpha \int_{\xi_{c}}^{\xi_{0}} f^{n} v^{2} d \xi
$$

where we have chosen signs so that $W>0$, and where all quantities are dimensionless (see text). Using equation (A5) to estimate the speed as a function of position, the work $W$ can be expressed as

$$
W=\alpha v_{0}^{2} \int_{\xi_{c}}^{\xi_{0}} f^{n} \mathrm{e}^{2 \beta\left(\xi-\xi_{0}\right)} d \xi
$$


where $\beta=\alpha\left\langle f^{n}\right\rangle$. If we make the simplifying approximation $f^{n} \approx 1 / 2$, the expression becomes

$$
W=\frac{1}{2} \alpha v_{0}^{2} \int_{\xi_{c}}^{\xi_{0}} \mathrm{e}^{\alpha\left(\xi-\xi_{0}\right)} d \xi,
$$

which can be integrated to yield

$$
W=\frac{1}{2} v_{0}^{2}\left[1-\mathrm{e}^{\alpha\left(\xi_{c}-\xi_{0}\right)}\right]=E_{0}\left[1-\mathrm{e}^{\alpha\left(\xi_{c}-\xi_{0}\right)}\right] .
$$

Conservation of energy implies $E_{f}=E_{0}-W$, so the remaining energy when these planets reach the core is given by

$$
E_{f}=\mathrm{e}^{\alpha\left(\xi_{c}-\xi_{0}\right)} E_{0} .
$$

Consider a giant planet with polytropic index $n=1$ and core radius $\xi_{c}=1$. The final energies $E_{f}$ predicted by equation (B5) for incoming rocky planets with masses $m_{\mathrm{p}}=1$, 10 , and $20 M_{\oplus}$ are given by $E_{f} / E_{0} \sim 0.03,0.20$, and 0.30 , in rough agreement with the numerically determined results.

\section{REFERENCES}

Abramowitz, M., \& Stegun, I. A., 1970, Handbook of Mathematical Functions (New York: Dover)

Adams, F. C., \& Bloch, A. M. 2009, ApJ, 701, 1381

Anic, A., Alibert, Y., \& Benz, W. 2007, A\&A, 466, 717

Arras, P., \& Socrates, A. 2010, ApJ, 714, 1

Artymowicz, P. 1993, ApJ, 419, 166

Baraffe, I., Chabrier, G., \& Barman, T. 2010, Reports on Progress in Physics, 73, 016901

Batygin, K., \& Stevenson, D. J. 2010, ApJ, 714, 238

Batygin, K., Stevenson, D. J., \& Bodenheimer, P. H. 2011, ApJ, 738, 1

Benvenuto, O. G., \& Brunini, A. 2008, Icarus, 195, 882

Bodenheimer, P., Laughlin, G., \& Lin, D.N.C. 2003, ApJ, 592, 555

Boley, A. C. 2009, ApJ, 695, 53

Boley, A. C., Helled, R., \& Payne, M. J. 2011, ApJ, 735, 30 
Borucki, W. J., Koch, D. G., Basri, G., et al. 2011, ApJ, 736, 19

Boss, A. P. 1997, Science, 276, 1836

Bramich, D. M., \& Horne, K. 2006, MNRAS, 367, 1677

Burrows, A. 2011, private communication

Burrows, A., Marley, M., Hubbard, W. B., Lunine, J. I., Guillot, T., Saumon, D., Freedman, R., Sudarsky, D., \& Sharp, C. 1997, ApJ, 491, 856

Burrows, A., Hubeny, I., Budaj, J., \& Hubbard, W. B. 2007, ApJ, 661, 502

Burrows, A., \& Orton, G. 2009, arXiv:0910.0248v1

Chandrasekhar, S. 1939, An Introduction to the Study of Stellar Structure (Chicago: University Chicago Press)

Crida, A., Sándor, Z., \& Kley, W. 2008, A\&A, 483, 325

Fortney, J. J., Saumon, D., Marley, M. S., Lodders, K., \& Freedman, R. S. 2006, ApJ, 642, 495

Gu, P., Bodenheimer, P. H., \& Lin, D.N.C. 2004, ApJ, 608, 1076

Guillot, T., \& Showman, A. P. 2002, A\&A, 385, 156

Helled, R., \& Shubert, G. 2009, ApJ, 697, 1256

Hubbard, W. B., Burrows, A. \& Lunine, J. I. 2002, ARA\&A, 40, 130

Ikoma, M., Guillot, T., Genda, H., Tanigawa, T., \& Ida S. 2006, ApJ, 650, 1150

Ketchum, J. A., Adams, F. C., \& Bloch, A. M. 2011a, ApJ, 726, 53

Ketchum, J. A., Adams, F. C., \& Bloch, A. M. 2011b, ApJ, 741, L2

Kley, W., Peitz, J., \& Bryden, G. 2004, A\&A, 414, 735

Korycansky, D. G., \& Zahnle, K. J. 2005, P\&SS, 53, 695

Laughlin, G., Crismani, M., \& Adams, F. C. 2011, ApJ, 729, L7

Li, S. L., Agnor, C. B., \& Lin, D.N.C. 2010, ApJ, 720, 1161

Mayor, M., \& Queloz, D. 1995, Nature, 378, 355 
Militzer, B., Hubbard, W. B., Vorberger, J., Tamblyn, I., \& Bonev, S. A. 2008, ApJ, 688, L45

Miller, N., \& Fortney, J. J. 2011, ApJ, 736, 29

Papaloizou, J.C.B., \& Terquem, C. 2006, Reports on Progress in Physics, 69, 119

Perna, R., Menou, K., \& Rauscher, E. 2010, ApJ, 724, 313

Phillips, A. C. 1994, The Physics of Stars (Chichester: Wiley)

Pollack, J. B., Hubickyj, O., Bodenheimer, P., Lissauer, J. J., Podolak, M., \& Greenzweig, Y. 1996, Icarus, 124, 62

Rauscher, E., \& Menou, K. 2010, ApJ, 745, 78

Sato, B. et. al. 2005, ApJ, 633, 465

Saumon, D., \& Guillot, T. 2004, ApJ, 609, 1170

Sudarsky, D., Burrows, A., \& Pinto, P. 2000, ApJ, 538, 885

Valencia, D., Sasselov, D. D., \& O'Connell, R. J. 2007, ApJ, 665, 1413

van Saders, J. L., \& Gaudi, B. S. 2011, ApJ, 729, 63

Ward, W. R. 1997, Icarus, 126, 261 